Sādhanā Vol. 39, Part 2, April 2014, pp. 391-407. (C) Indian Academy of Sciences

\title{
Two-motor single-inverter field-oriented induction machine drive dynamic performance
}

\author{
BHAKTI M JOSHI* and MUKUL C CHANDORKAR \\ Department of Electrical Engineering, Indian Institute of Technology Bombay, \\ Powai, Mumbai 400 076, India \\ e-mail: bmjoshi@ee.iitb.ac.in; mukul@ee.iitb.ac.in
}

MS received 22 February 2013; revised 19 September 2013; accepted 7 October 2013

\begin{abstract}
Multi-machine, single-inverter induction motor drives are attractive in situations in which all machines are of similar ratings, and operate at approximately the same load torques. The advantages include small size compared to multi-inverter system, lower weight and overall cost. However, field oriented control of such drives is a challenge since no two motors will have exactly the same operating conditions at any time. In general, at least some motors in the system will operate away from perfect field orientation. It is therefore important to analyse their torque dynamics carefully. This paper discusses existing multi-machine field-oriented control methods, and analyses and compares them from the viewpoint of dynamic performance. For this, an analytical framework based on small-signal analysis is developed to compare multi-machine control methods. This analysis is verified by simulations and experiments.
\end{abstract}

Keywords. Multi-machine induction motor drives; field oriented control dynamics; small-signal analysis.

\section{Introduction}

Multi-motor induction machine drives are widely used for applications such as paper and steel rolling mills (Ando et al 2004; Inoue et al 2011), conveyer systems (Mohktari \& Alizadeh 2007), and electric propulsion systems like electric traction, ship propulsion and electric vehicles (Kelecy \& Lorenz 1994; Escané et al 1999; Ruxi et al 2006). For multi-motor drives, the drive system structure and control method depends on the application for which they are used and also on the performance, speed and accuracy needed from the drive system. For applications which do not demand a very high dynamic performance, a multi-motor, multi-inverter drive system using a modified $V / f$ control strategy is described in (Iyer et al 2011). Another control method based on $V / f$ strategy is given for a space vector modulated CSI drive in (Ma et al 2001) for medium voltage multi-motor drives.

*For correspondence 


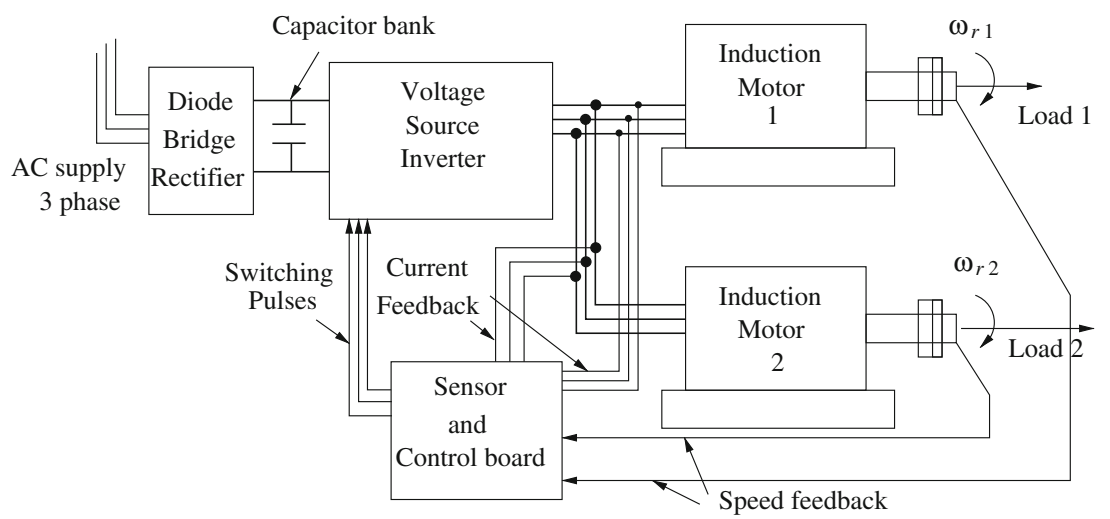

Figure 1. A typical single-inverter two-machine drive system.

For applications such as paper mills, drives with synchronized speeds are needed. For this, electric drives comprising multi-motor, multi-inverter structures are more flexible and reliable than those with mechanical transmission systems like line-shaft, pulleys and gears. Since the motor speeds need to be equal under all load conditions, each motor needs a separate converter for such systems (Turl et al 2002). Use of separate inverters to drive an adaptive electric differential based on a dual induction motor configuration is given in (Tabbache et al 2011). An electrical differential consisting of two induction machine drives with separate inverters controlled with Direct Torque Control (DTC), is discussed in (Hartani et al 2008). A systematic survey of multi-motor drives based on the method of coupling and mutual influence is presented in (Jeftenic et al 2006), in which, drives are classified based on the degree of mechanical coupling between the machines. Crelerot et al (1993) present a study of an electrical differential motor which consists of a common stator and two rotors, for an electric car application.

For applications where the requirements for speed synchronization are not stringent, a drive system consisting of multiple machines fed by a single inverter offers an attractive solution due to the reduction in cost, size and power electronic components. Electric traction is one application where such multi-machine, mono-inverter drives can be effectively used (Escané et al 1999, 2000; Matsumoto et al 2001). A simple drive train design (due to the absence of gears) is also an added advantage of such a drive system (Kelecy \& Lorenz 1994). Different modelling techniques have been used for the multi-machine drives. A graphical modelling approach is used to model a parallely connected multi-machine system in (Penã-Eguiluz et al 2002), whereas global modelling approach is used in (Bouscayrol et al 2000) for multi-machine systems. A formulation of multi-machine, multi-converter systems using several couplings is given in (Bouscayrol et al 2005).

Drives comprising multi-phase, multi-motor induction machines fed by a single inverter are studied in detail (Levi et al 2004, 2007). Due to additional degrees of freedom available in such machines, an independent control can be achieved for each machine with series or parallel connection. The stator windings of two or more multi-phase machines can be connected in series with an appropriate phase transposition so that the currents which produce rotating mmf in one machine, do not produce rotating $\mathrm{mmf}$ in other machines and vice versa (Levi et al 2004). In case of three-phase machines, lack of additional degrees of freedom does not allow series connection. In this paper, we consider a parallely connected two-machine three-phase induction motor drive fed by a single inverter. Figure 1 shows such a typical two-machine drive system 
(Joshi \& Chandorkar 2011). Here, current and speed feedback is taken from both machines which are fed by a single voltage source inverter for calculating the reference voltages to be applied to the machines.

This paper is concerned with the dynamic performance of two-motor single-inverter drive systems in which the motors are identical. However, differences in their mechanical loads prevent both motors from being field-oriented simultaneously. Despite this, it is important for the motors to have good dynamic torque performance. A good torque performance is needed for the successful implementation of power failure ride-through (Joshi \& Chandorkar 2012), and several other drive features such as load-dependent magnetic flux reduction to enhance efficiency (Taylor 1994; Kazmierkowski 1997).

To obtain a good dynamic torque response from the drive, high performance induction machine control strategies such as Field Oriented Control (FOC) (Kelecy \& Lorenze1994; Matsuse et al 2002; Levi et al 2004, 2007; Joshi et al 2011; Joshi \& Chandorkar 2012) and Direct Torque Control (DTC) (Escané et al 1999, 2000; Mohktari \& Alizadeh 2007; Joshi $\&$ Chandorkar 2011) can be used to control two-machine, single-inverter systems. High performance multi-motor control schemes discussed in the literature can be broadly classified as the Mean control and the Master-Slave control. Both of these can be considered as special cases of controlling a weighted-average of the machine quantities with the weights equal to $0.5,0.5$ for the Mean control and 1, 0 for the Master-Slave control, 1 for the machine which is controlled and 0 for the machine which is connected in parallel with the former. These machines are referred to as primary and secondary machines respectively, throughout this paper.

Dynamic performance of multi-motor control methods based on Mean and Master-Slave field oriented control is the primary focus of this paper. We present a framework in which to analyse the dynamic performance based on small-signal analysis. This is validated by simulation and experiments. Small-signal analysis of single-machine transients is well known (Novotny \& Lipo 1996). In this paper, we extend the method to study multi-machine drive systems by defining and evaluating transfer functions between the machine torques, and the machine currents in a field-oriented control scenario. We apply this analysis to motors of different ratings and show how dynamic performance is quantitatively linked to differences in machine load torque for the multi-machine system. We concentrate on a two-machine system. Based on analysis, simulation and experiments, we conclude that the Mean control method has better overall dynamic torque performance than the Master-Slave field-oriented control for a given reference speed for the drive and load torques in smaller machines. Both methods have similar behaviour for the larger machines.

\section{Comparison of control strategies}

MS and Mean control are the two broadly used control methods for multi-machine, singleinverter induction motor drives. Figures 2 and 3 (Joshi \& Chandorkar 2012) illustrate the Master-Slave (MS) and the Mean (average) control strategies, respectively. The superscript $e$ represents the quantities in the synchronous reference frame. In case of machines with equal rating, parameter values and load torque, both strategies are essentially equivalent to two singlemotor, single-inverter induction machine drives. However, during normal drive operation, the loads on both machines may not always remain exactly equal. For example, in a traction system, a change in the surface condition between one of the wheel-rail surfaces will cause a change in the coefficient of friction between them, in turn causing a change in the load torque on the motor 


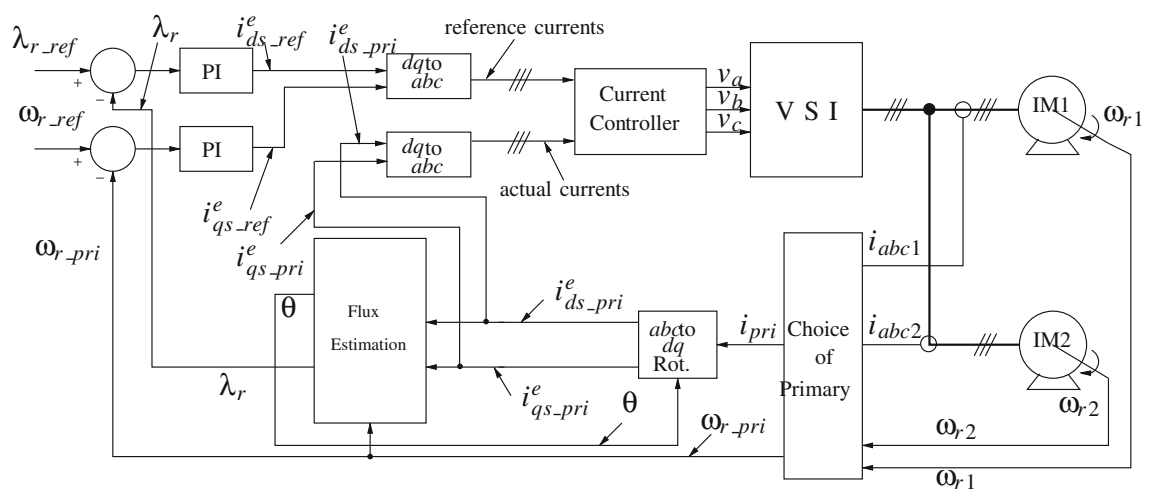

Figure 2. Multimachine FOC: MS control.

coupled to that particular wheel. In case of different loads on the machines, each of these strategies shows different dynamic behaviour as further discussed in detail in this paper. In speed controlled/regulated drives, a change in the reference torque is caused by either the change in the load or reference speed via the operation of the speed controller. However, in most cases, the speed control loop can not act very fast owing to the inertia of the machines and load. Since it is our intention to study the torque dynamics of the drives which are much faster in nature, the outer speed control loop is not considered in the analysis. The loads on the machines are assumed to be constant and the speed response of the drive is not studied, the focus being on the torque response.

\subsection{Master-Slave (MS) strategy}

As shown in figure 2 (Joshi \& Chandorkar 2012), in this strategy, only one machine is controlled using the conventional FOC referred here as the Primary machine, while the other machine is connected in parallel with the Primary. The suffixes pri and sec are used for the quantities corresponding to the Primary and Secondary machines, respectively. The choice of the Primary machine is governed by the magnitude of rotor flux linkages which in turn depends on the load

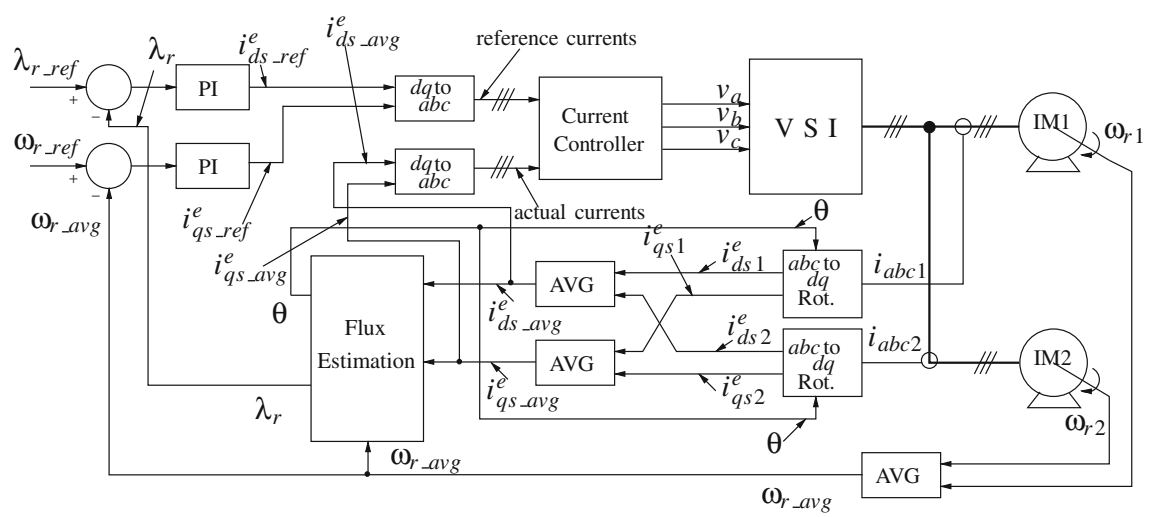

Figure 3. Multimachine FOC: Mean control. 
torque on the machine. For the machine which is lightly loaded, the magnitude of rotor flux linkages is greater than that for the heavily loaded machine, since the voltage drop across the rotor resistance $\left(i \times R_{r}\right)$ is larger for a machine negotiating a larger load. If the lightly loaded machine is chosen as the Primary machine, the rotor flux will be controlled and made equal to the rated value for that machine, avoiding saturation. A current feedback taken from the Secondary machine as well so as to facilitate the choice of Primary. However, feedback from the Secondary machine is not used by the controller to decide the reference variables. A speed feedback is taken from both machines.

Since both machines are of identical rating and parameter values, equal load torque on them will result in both machines being field orientated. However, if the Secondary machine load changes, it loses its field orientation and its dynamic performance deteriorates. Figure 4 a shows the torque dynamics of both machines when the drive is controlled using the MS strategy. The torque response of both machines to a step change in $i_{q s \_r e f}^{e}$ is plotted. It is observed that the Primary machine torque ( $T_{e m \_}$pri $)$closely follows the reference command, whereas the Secondary machine torque $\left(T_{e m \_s e c}\right)$ shows an oscillatory and delayed response.

\subsection{Mean strategy}

As illustrated in figure 3 (Joshi \& Chandorkar 2012), in this strategy, the average of currents and speed is calculated and is controlled in the classical field oriented manner, assuming that they are the quantities sensed from a fictitious average machine. The subscript avg is used to denote the average quantities. When the load torques on both machines are different, controlling the average quantities may cause the rotor flux magnitude of one machine (lightly loaded) to increase beyond the rated value. Since neither of the two machines is controlled directly, both machines are away from the perfect field orientation. This results in a deteriorated dynamic performance for both machines. Figure $4 \mathrm{~b}$ shows the torque dynamics of both machines controlled using the Mean strategy. It is observed that the torque dynamics of both machines $\left(T_{e m \_1}\right.$ and $\left.T_{e m \_}\right)$show an oscillatory and delayed response. In both of these strategies discussed, it is clear that the difference in the load torques on the two machines is the reason behind the dynamic performance deterioration of one (in MS strategy) or both (in Mean strategy) machines. A detailed analysis of this phenomenon is presented in the next sections. The essential difference between the dynamics of the two control methods is that in the MS method, the condition of the Primary machine affects the Secondary machine, but the reverse is not true. In the Mean method, both machines affect each other.

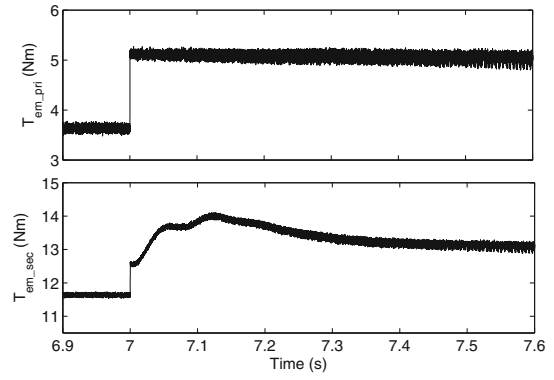

(a) MS strategy
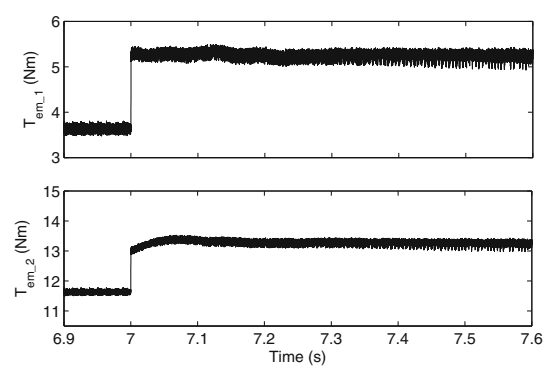

(b) Mean strategy

Figure 4. Multimachine dynamics: Simulation plots. 


\section{System modelling}

In this section, as a first step towards analysing the dynamic behaviour of the system, the drive system is modelled in terms of a set of differential equations.

\subsection{Large signal induction machine model}

Induction machine dynamics are generally represented by a fifth-order nonlinear system of differential equations in $d-q$ coordinates in the synchronous reference frame (1) and (2) (Vas 1998; Krause et al 2002).

$$
\begin{aligned}
\frac{d}{d t}\left[\begin{array}{c}
i_{q s}^{e} \\
i_{d s}^{e} \\
\lambda_{q r}^{e} \\
\lambda_{d r}^{e}
\end{array}\right]=\left[\begin{array}{cccc}
-\gamma & -\omega_{e} & \alpha \beta & -\beta \omega_{r} \\
\omega_{e} & -\gamma & \beta \omega_{r} & \alpha \beta \\
\alpha M & 0 & -\alpha & -\left(\omega_{e}-\omega_{r}\right) \\
0 & \alpha M & \left(\omega_{e}-\omega_{r}\right) & -\alpha
\end{array}\right]\left[\begin{array}{c}
i_{q s}^{e} \\
i_{d s}^{e} \\
\lambda_{q r}^{e} \\
\lambda_{d r}^{e}
\end{array}\right]+\frac{1}{\sigma}\left[\begin{array}{c}
v_{q s}^{e} \\
v_{d s}^{e} \\
0 \\
0
\end{array}\right] \\
\frac{d}{d t} \omega_{r}=\left(\frac{1}{J}\right)\left(T_{e m}-T_{L}\right), \text { where, } \\
T_{e m}=\left(\frac{3 P M}{4 L_{r}}\right)\left(\lambda_{d r}^{e} i_{q s}^{e}-\lambda_{q r}^{e} i_{d s}^{e}\right) .
\end{aligned}
$$

In this, $T_{e m}$ is the electromagnetic torque produced by the machine and $T_{L}$ is the load torque. The definitions of the constants used here are given in (3), where, $R_{S}$ and $R_{r}$ are stator and rotor resistances, respectively, $L_{l s}$ and $L_{l r}$ are leakage inductances for stator and rotor respectively, and $L_{s}=L_{l s}+M$ and $L_{r}=L_{l r}+M$, where, $M$ is the magnetizing inductance. $J$ is the combined moment of inertia of motor and load and $P$ is the number of poles. $i_{q s}^{e}, i_{d s}^{e}$ are the $q$ and $d$ axis stator currents respectively, and $\lambda_{q r}^{e}$ and $\lambda_{d r}^{e}$ are the $q$ and $d$ rotor flux linkages respectively. $\omega_{e}$ and $\omega_{r}$ are the synchronous and rotor speeds, respectively.

$$
\begin{gathered}
\alpha=\frac{R_{r}}{L_{r}}, \sigma=L_{s}-\frac{M^{2}}{L_{r}}, \beta=\frac{M}{\sigma L_{r}}, \\
\mu=\left(\frac{3}{2}\right)\left(\frac{P}{2}\right)\left(\frac{M}{J L_{r}}\right), \gamma=\frac{M^{2} R_{r}}{\sigma L_{r}^{2}}+\frac{R_{s}}{\sigma} .
\end{gathered}
$$

The presence of two induction motors, each of which is a coupled, nonlinear system of equations in itself, makes the two-machine, single-inverter system difficult to analyse when the dynamic performance is of the concern. However, an insight into the dynamic performance of the twomachine system can be obtained by considering a linearized system model. Hence, to facilitate the analysis, the nonlinear system of equations is small-signal linearized around an operating point, the subsequent details of which are given below. In the analysis, our aim is to model the effect of deviation from field orientation in the multi-machine drive system. To simplify the analysis without losing much generality, we assume that the drive current controller is fast enough, so that the actual motor currents are always equal to their reference values.

\subsection{Small-signal modelling: MS strategy}

The Primary machine in MS control is always field-oriented. Its current control determines the voltage that the inverter applies commonly to both machines. Hence the interest here is only in the dynamics of the Secondary machine, which is away from field orientation by an amount that 
depends on the applied voltage and its load torque. After the small-signal linearization of the drive system, the resultant linear system of equations can be represented in the following form (Krause et al 2002):

$$
\begin{aligned}
\frac{d}{d t}(\Delta x(t)) & =A \Delta x(t)+B \Delta u(t) \\
\Delta y(t) & =C \Delta x(t)+D \Delta u(t),
\end{aligned}
$$

where $\Delta x(t)=\left[\Delta x_{1} \Delta x_{2} \ldots \Delta x_{n}\right]$ is a vector of small-signal state variables, $\Delta u(t)$ and $\Delta y(t)$ are the vectors of small-signal input and output variables, respectively.

As discussed earlier, in the MS strategy, the Primary machine currents are controlled and are assumed to be equal to their reference values. As a result, the corresponding small-signal currents are also equal to the small-signal reference values. Also, the currents are controlled so as to achieve field orientation in the Primary machine. For a field oriented machine, the $d$ axis flux linkages are maintained at the rated flux and the $q$ axis flux linkages are zero. Since both $d$ and $q$ axes flux linkages remain unchanged, the corresponding small-signal variables are zero. Summing up, the following relations (5) are valid for the Primary machine small-signal variables.

$$
\begin{aligned}
\Delta i_{d s_{-} p r i}^{e} & =\Delta i_{d_{-} r e f} \\
\Delta i_{q s_{-} p r i}^{e} & =\Delta i_{q_{-} r e f} \\
\Delta \lambda_{d r_{-} p r i}^{e} & =0 \quad\left(\because \lambda_{d r_{-} p r i}^{e}=\lambda_{r_{-} r e f}\right) \\
\Delta \lambda_{q r_{-} p r i}^{e} & =0 .
\end{aligned}
$$

The small-signal dynamic equations of the Primary machine are obtained by substituting from (5) in the small-signal linearized induction machine model equations. The synchronous speed $\omega_{e}$ is obtained by adding the rotor speed of the Primary machine to the calculated slip speed using the Primary machine variables (according to the classical field orientation relation) as given in (6) (Vas 1998). This value of $\omega_{e}$ is substituted in the Secondary machine small-signal linearized model equations as well. The vectors of small-signal state and input variables are as given in (7) (the superscript $T$ represents transpose of the vector). $\Delta x_{p r i}(t)$ and $\Delta x_{s e c}(t)$ are the vectors of small-signal state variables corresponding to the Primary and the Secondary machines, respectively. It can be noted that $\Delta x_{\text {pri }}(t)$ has fewer entries than $\Delta x_{s e c}(t)$. As mentioned earlier, $\Delta T_{L 1}$ and $\Delta T_{L 2}$ are considered as the disturbance inputs for the system and are taken to be equal to zero for this analysis.

$$
\begin{aligned}
& \omega_{e}=\omega_{r_{-} p r i}+\frac{\alpha M i_{q s_{-} p r i}^{e}}{\lambda_{d r_{-} p r i}^{e}} \\
& \Delta x(t)=\left[\begin{array}{ll}
\Delta x_{p r i}(t) & \Delta x_{\text {sec }}(t)
\end{array}\right]^{T}, \text { where } \\
& \Delta x_{p r i}(t)=\left[\begin{array}{lll}
\Delta i_{d s_{-} p r i}^{e} & \Delta i_{q s_{-} p r i}^{e} \Delta \omega_{r_{-} p r i}
\end{array}\right]^{T} \\
& \Delta x_{s e c}(t)=\left[\begin{array}{llll}
\Delta i_{d s_{-} s e c}^{e} & \Delta i_{q s_{-} s e c}^{e} & \Delta \lambda_{d r_{-} s e c}^{e} \Delta \lambda_{q r_{-} s e c}^{e} \Delta \omega_{r_{-} s e c}
\end{array}\right]^{T} \\
& \Delta u(t)=\left[\begin{array}{ll}
\Delta i_{d_{-}} \text {ref } & \Delta i_{q_{-} r e f}
\end{array}\right]^{T} .
\end{aligned}
$$

\subsection{Small signal modelling: Mean strategy}

As shown in figure 3, the average values of speed and currents are calculated and they are controlled in the classical field oriented manner. Suffixes 1 and 2, are used to denote the quantities corresponding to machine 1 and 2, respectively. As mentioned earlier, the average currents 
$i_{d s_{-} a v g}^{e}$ and $i_{q s_{-} a v g}^{e}$ are assumed to be the stator currents corresponding to a virtual average machine, which is controlled with FOC, and has a speed $\omega_{r_{-} a v g}$. Since this mean machine is field oriented, $i_{d s \_a v g}^{e}$ controls the rotor flux for this machine $\left(\bar{\lambda}_{m}\right)$ in a completely decoupled manner. Here, $\lambda_{m}$ denotes the rotor flux linkages for the mean machine, perfectly oriented along the synchronous frame $d$ axis. However, since none of the two machines are actually field oriented, their rotor flux linkages are different from $\lambda_{m}$. Similar to the MS strategy, hysteresis current control is used to control the mean machine currents. Thus it can be safely assumed that the mean machine currents $i_{d s_{\_} a v g}^{e}$ and $i_{q s_{-} a v g}^{e}$ are equal to the reference values $i_{d_{-} r e f}$ and $i_{q_{-} r e f}$, respectively. As a result, the small-signal mean machine currents are also equal to the small-signal reference values. Summing up, the following relations (8) are valid for the mean machine smallsignal variables. The synchronous speed is obtained by adding the average rotor speed to the slip speed calculated by using the average current and flux values and is as given by (9). The vector of small-signal state variables is as given in (10). The input variables are the same as those given for MS control in (7).

$$
\begin{aligned}
& \Delta i_{d s_{-} a v g}^{e}=\Delta i_{d \_r e f} \\
& \Delta i_{q s \_a v g}^{e}=\Delta i_{q \_r e f} \\
& \Delta \lambda_{m}=0 \quad\left(\because \lambda_{m}=\lambda_{r_{-} r e f}\right) \\
& \omega_{e}=\omega_{r \_a v g}+\frac{\alpha M i_{q s \_a v g}^{e}}{\lambda_{m}} \\
& \Delta x(t)=\left[\begin{array}{ll}
\Delta x_{1}(t) & \Delta x_{2}(t)
\end{array}\right]^{T}, \quad \text { where } \\
& \Delta x_{1}(t)=\left[\begin{array}{lllll}
\Delta i_{d s_{-} 1}^{e} & \Delta i_{q s_{-} 1}^{e} & \Delta \lambda_{d r_{-} 1}^{e} & \Delta \lambda_{q r_{-} 1}^{e} & \Delta \omega_{r_{-} 1}
\end{array}\right]^{T}
\end{aligned}
$$

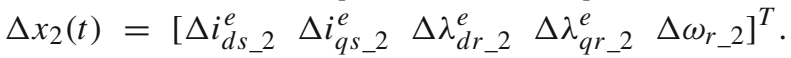

\section{Small-signal transfer function}

Model equations obtained from small-signal linearization of the drive system facilitate their representation in matrix form and operations such as simultaneous solving of equations to eliminate certain variables. In order to study the nature of dynamic response of the machines, the torque response of individual machines to a fast change in the $q$ axis current reference is of interest to us, while the $d$ axis current reference is maintained at a constant (rated) value. Hence, we represent the small-signal linearized electromagnetic torque $\left(\Delta T_{e m}\right)$ in terms of the reference value $\Delta i_{q \_} r e f$. The small signal linearized model equations of the system as developed in the previous section are used to calculate these transfer functions as described below.

\subsection{MS control}

For the MS control, the Primary machine is field oriented and hence its torque response follows the $q$ axis current reference almost instantaneously. Hence for the Primary machine, the transfer function (and also the small signal transfer function $G_{p r i}$ ) which relates the torque to the $q$ axis current reference is essentially a constant and can be calculated as given by (11).

$$
\frac{T_{e m_{-} p r i}(s)}{i_{q_{-} r e f}(s)}\left(=\frac{\Delta T_{\text {em_pri }}}{\Delta i_{q_{-} r e f}}\right)=\left(\frac{3 P M}{4 L_{r}}\right) \lambda_{r_{-} r e f}=G_{\text {pri }} .
$$


When the load torques on the two machines are different, the Secondary machine is not in a state of perfect field orientation. To be able to find out the small signal transfer function relating $\Delta T_{\text {em_sec }}$ to $\Delta i_{q_{-} \text {ref }}$, it is needed to represent the Secondary machine state variables in terms of $\Delta i_{q \_} r e f$, which is done as follows. Small-signal differential equations for Secondary machine rotor flux are as given by (12) and (13), in which, $\omega_{e}$ is the synchronous speed given by (6). Small-signal linearization of (6) followed by substitutions in (5) results in (14).

$$
\begin{aligned}
& \frac{\Delta \lambda_{d r_{-} s e c}^{e}}{d t}= \alpha M \Delta i_{d s_{-} s e c}^{e}+\left(\omega_{e 0}-\omega_{r_{-} s e c}\right) \Delta \lambda_{q r_{-} s e c}^{e} \\
&+\lambda_{q r_{-} s e c 0}^{e}\left(\Delta \omega_{e}-\Delta \omega_{r_{-} s e c}\right)-\alpha \Delta \lambda_{d r_{-} s e c}^{e} \\
& \frac{\Delta \lambda_{q r_{-} s e c}^{e}}{d t}= \alpha M \Delta i_{q s_{-} s e c}^{e}-\left(\omega_{e 0}-\omega_{r_{-} s e c 0}\right) \Delta \lambda_{d r_{-} s e c}^{e} \\
&-\lambda_{d r_{-} s e c 0}^{e}\left(\Delta \omega_{e}-\Delta \omega_{r_{-} s e c}\right)-\alpha \Delta \lambda_{q r_{-} s e c}^{e} \\
& \Delta \omega_{e}=\Delta \omega_{r_{-} p r i}+\left(\alpha M / \lambda_{r_{-} r e f}\right) \Delta i_{q_{-} r e f} .
\end{aligned}
$$

Small-signal differential equations for the rotor speed of the Primary and Secondary machines are given by (15)-(16) respectively.

$$
\begin{aligned}
\frac{\Delta \omega_{r_{-} p r i}}{d t}= & \left(K_{t} / J\right) \lambda_{d r_{-} p r i 0}^{e} \Delta i_{q_{-} r e f} \\
\frac{\Delta \omega_{r_{-} s e c}}{d t}= & \left(\lambda_{d r_{-} \sec 0} \Delta i_{q s_{-} s e c}^{e}-\lambda_{q r_{-} s e c 0} \Delta i_{d s_{-} s e c}^{e}\right. \\
& \left.+i_{q s_{-} \sec 0}^{e} \Delta \lambda_{d r_{-} s e c}^{e}-i_{d s_{-} s e c 0}^{e} \Delta \lambda_{q r_{-} s e c}^{e}\right) .
\end{aligned}
$$

Substituting from (15) and (16) in (12) and (13) followed by solving the flux equations simultaneously, we can represent small-signal flux variables in terms of $\Delta i_{d s_{-} s e c}^{e}, \Delta i_{q s_{-} s e c}^{e}$ and $\Delta i_{q_{\_} r e f}$. To be able to represent $\Delta i_{d s_{-} s e c}^{e}, \Delta i_{q s_{-} s e c}^{e}$ in terms of $\Delta i_{q \_r e f}$, Secondary machine stator voltage equations are small-signal linearized, and substitutions are made for the speed and flux smallsignal variables. Simultaneous solving of the resultant equations gives us the Secondary machine currents in terms of $\Delta i_{q_{-} r e f}$. After this step, we essentially have all the state variables of the Secondary machine in terms of $\Delta i_{q_{-} r e f}$ and we arrive at (16), where $G_{s e c}$ is the desired transfer function.

$$
\frac{\Delta T_{\text {em_sec }}(s)}{\Delta i_{q_{-} \text {ref }}(s)}=G_{\text {sec }}(s) .
$$

We use a symbolic mathematics package to find out $G_{s e c}$ and to determine its step response. It is observed that, when the loads on the machines are equal, the transfer function $G_{s e c}$ is equal to $G_{\text {pri }}$, thus indicating that both machines are field oriented in equal load torque situation.

\subsection{Mean control}

For the mean control, the average values of $d$ and $q$ axis currents and speeds are controlled to make the virtual average machine field oriented. When there is a difference in the loads on the individual machines, neither of the two machines is field oriented. Hence in the case of mean control, it is important to study the torque dynamics of both machines unlike the case of MS control, where only the Secondary machine is affected by the load difference. 


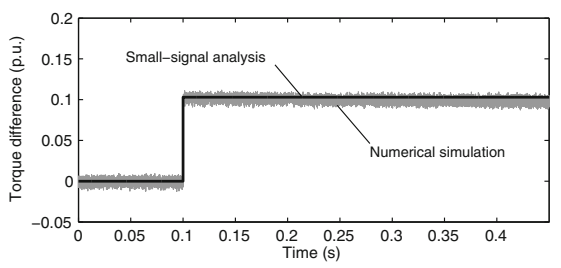

(a) Primary machine

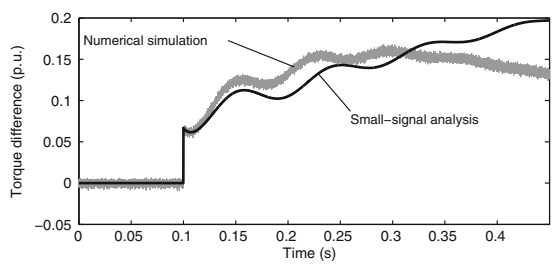

(b) Secondary machine

Figure 5. Torque dynamics in MS control: $3.73 \mathrm{~kW}$ machines.

To find the small-signal transfer functions which relate the electromagnetic torques of both machines to $\Delta i_{q_{-} \text {ref }}$, essentially a similar procedure is followed as done for the MS control (with conditions given by (8) and (9)), however in this case, the substitutions are made in the torque equations of both machines. The final torque expressions are given by (17) and (18). In this equation, $G_{1}(s)$ and $G_{2}(s)$ are the small-signal transfer functions of interest. As done for the analysis of MS control, we use a symbolic mathematics package to evaluate the transfer functions and the step response.

$$
\begin{aligned}
& \frac{\Delta T_{\text {em_1 } 1}(s)}{\Delta i_{q_{-} r e f}(s)}=G_{1}(s), \\
& \frac{\Delta T_{e m_{-} 2}(s)}{\Delta i_{q \_r e f}(s)}=G_{2}(s) \text {. }
\end{aligned}
$$

\section{Torque dynamics using small-signal transfer function}

The small-signal transfer functions which relate the electromagnetic torque to $\Delta i_{q \_} r e f$ can be used to study the torque dynamics as a result of a step change in $\Delta i_{q \_}$ref. To obtain the smallsignal response of the system, a step change of $10 \%$ of the rated $q$ axis current is given in the numerical simulation of the drive. Since the small-signal variables represent the change in the operating point from its initial state, the small-signal transfer function essentially gives the change in the torque from its initial state. Hence in the simulations, the corresponding change in the torque is found out by subtracting the initial value of electromagnetic torque from the value after the step change in the reference. The torque difference thus obtained is divided by its base value to convert it in per unit. The p.u. torque responses thus obtained by numerical simulation and by calculating the step response of the small-signal transfer function are superimposed on the same time scale. Figure 5 shows such plots for the Primary and Secondary machines $(3.73 \mathrm{~kW})$ controlled by the MS strategy whereas figure 6 shows the dynamic response for both machines

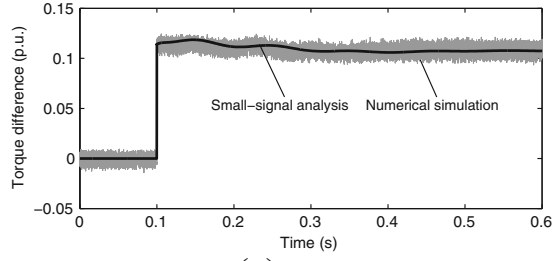

(a) Machine 1

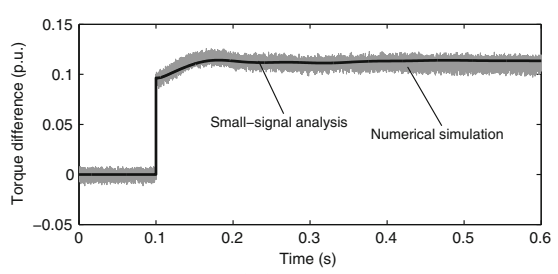

(b) Machine 2

Figure 6. Torque dynamics in mean control: $3.73 \mathrm{~kW}$ machines. 


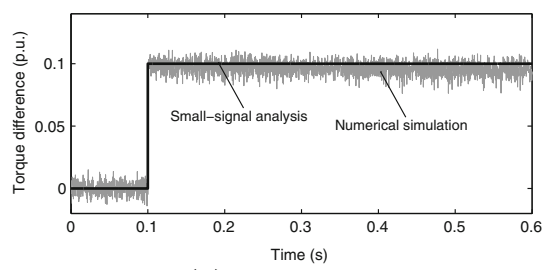

(a) Primary machine

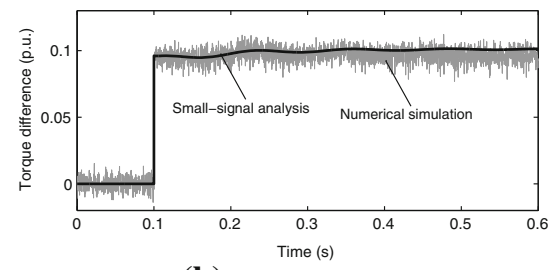

(b) Secondary machine

Figure 7. Torque dynamics in MS control: $37.3 \mathrm{~kW}$ machines.

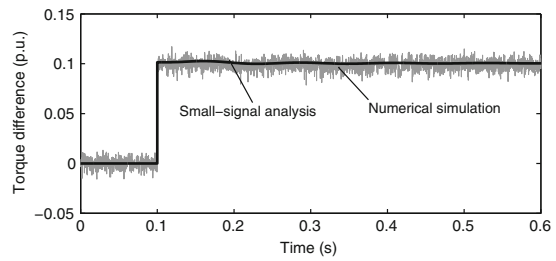

(a) Machine 1

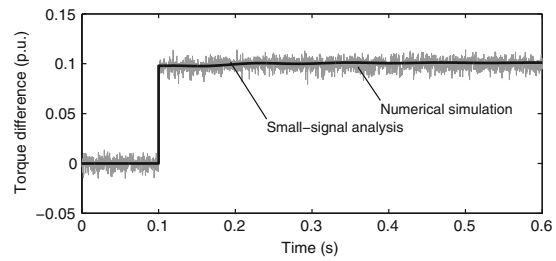

(b) Machine 2

Figure 8. Torque dynamics in mean control: $37.3 \mathrm{~kW}$ machines.

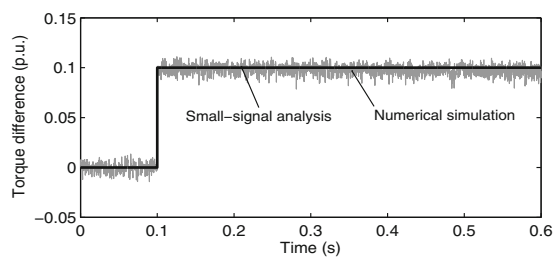

(a) Primary machine

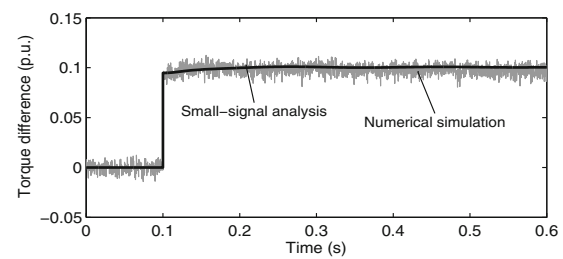

(b) Secondary machine

Figure 9. Torque dynamics in MS control: $373 \mathrm{~kW}$ machines.

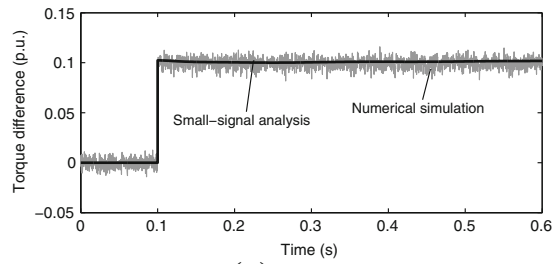

(a) Machine 1

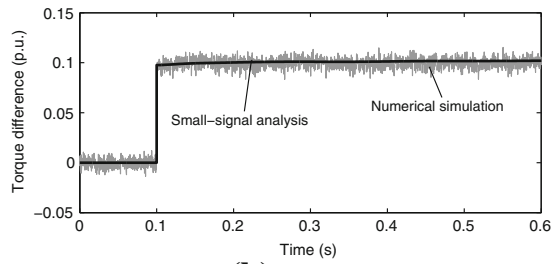

(b) Machine 2

Figure 10. Torque dynamics in mean control: $373 \mathrm{~kW}$ machines. 
Table 1. Induction motor parameters. Parameter values and machine ratings (all 3 phase, $50 \mathrm{~Hz}, 4$ pole).

\begin{tabular}{lccc}
\hline Rating $(\mathrm{kW})$ & 3.73 & 37.3 & 373.0 \\
Voltage $(\mathrm{V})$ & 415 & 460 & 2300 \\
$\mathrm{R}_{s}(\Omega)$ & 4.9 & 0.087 & 0.262 \\
$\mathrm{R}_{r}(\Omega)$ & 8.1 & 0.228 & 0.187 \\
$\mathrm{~L}_{l s}(\mathrm{mH})$ & 32.15 & 0.8 & 3.3 \\
$\mathrm{~L}_{l r}(\mathrm{mH})$ & 32.15 & 0.8 & 3.25 \\
$\mathrm{M}(\mathrm{mH})$ & 809.6 & 34.7 & 143.2 \\
$\mathrm{~J}\left(\mathrm{~kg}-\mathrm{m}^{2}\right)$ & 0.013 & 1.662 & 11.06 \\
\hline
\end{tabular}

controlled by the Mean strategy. Similarly, the plots for $37.3 \mathrm{~kW}$ machines for the MS and Mean control are shown in figures 7 and 8, respectively. Figures 9 and 10 show the plots for $373 \mathrm{~kW}$ machines (initial torque values for the machines are 0.25 p.u and 0.8 p.u. and the reference speed is 0.25 p.u. for all cases). The machine ratings and parameters are as given in table 1 . It can be observed from the figures that, for the MS control, the plots obtained from numerical simulations and the step response of the small-signal transfer function match for the Primary machine. For the Secondary machine, there is a close match between both plots for $37.3 \mathrm{~kW}$ and $373 \mathrm{~kW}$ machines, which also validates the analysis. However, for $3.73 \mathrm{~kW}$ machine, the plots are observed to deviate from each other as the time progresses. The reason for this behaviour is the low inertia of the $3.73 \mathrm{~kW}$ machines, because of which the speed increases significantly after a step change in the torque reference. This change in speed is no more a small-signal quantity. This effect is not significant for higher rated machines owing to their larger inertia. This fact was further verified by increasing the machine inertia for the $3.73 \mathrm{~kW}$ machines and observing its effect on the plots. The corresponding plots are given in figure 11, where the inertia is increased 5 times the original value. It can be observed that the plots match more closely, since the increased inertia reduces the rate of change of speed.

The information about the nature of small-signal transfer function dynamic response is contained in the transfer function poles (eigenvalues) and zeroes as discussed below.

\subsection{MS control}

Partial fraction expansion of $G_{s e c}$ results in 5 terms which are functions of $s$ (i.e., 5 poles and 5 residues) and a direct term $k_{s e c}$. These 5 eigenvalues are related to the Secondary machine states and can be identified as stator, rotor and speed eigenvalues (Krause et al 2002). Response of the pole pair with larger real part magnitude decays fast and they can be identified as the stator

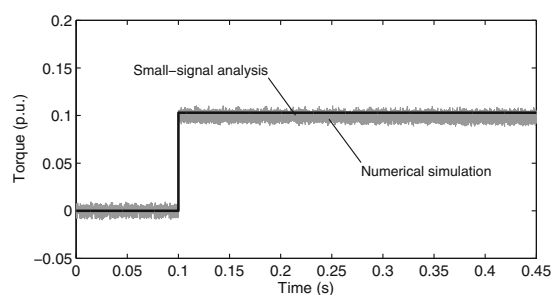

(a) Primary machine

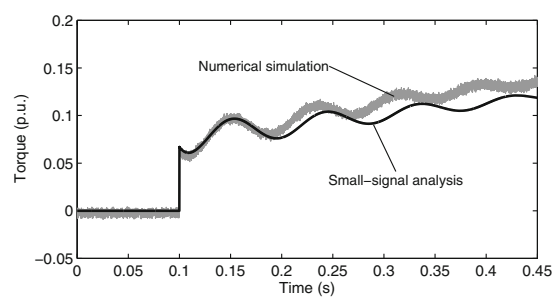

(b) Secondary machine

Figure 11. Torque dynamics in MS control: $3.73 \mathrm{~kW}$ machines for increased inertia. 


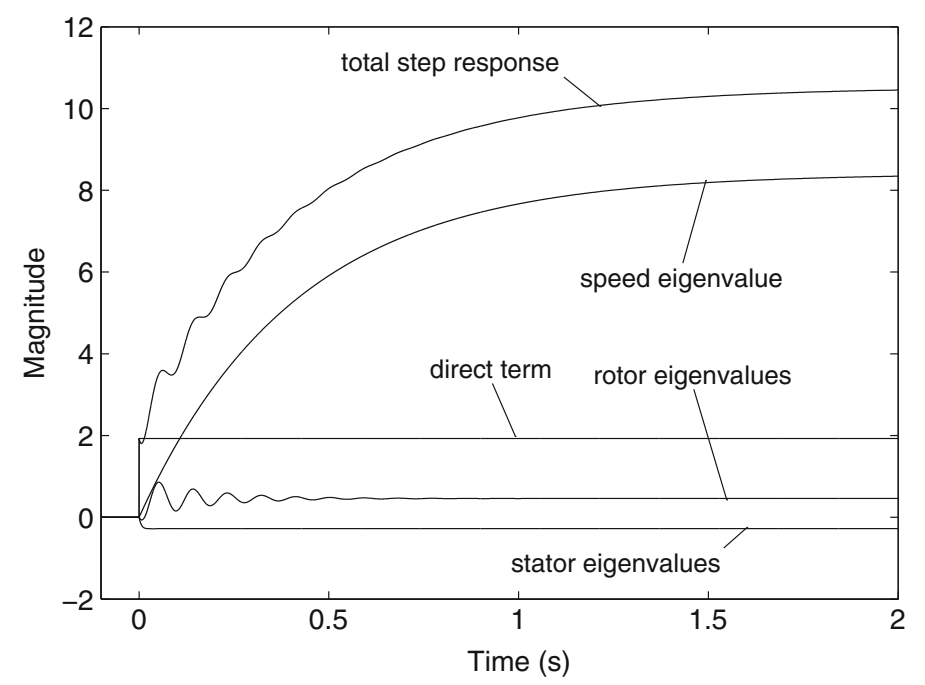

Figure 12. Components of $G_{\text {sec }}$ for $3.73 \mathrm{~kW}$ machines.

eigenvalues. The second pole pair is relatively slow decaying and is responsible for the visible oscillations in the torque response, and can be identified as rotor eigenvalues, and the real pole as the speed eigenvalue.

\subsection{Mean control}

For the Mean control, $G_{1}$ and $G_{2}$ both have 7 poles which are equal for both transfer functions. $G_{1}$ and $G_{2}$ each have 7 residues and a direct term each (denoted by $k_{1}$ and $k_{2}$, respectively). For the Mean strategy, there are 10 state variables as given in (10), however the stator currents and speeds are related algebraically since their average values are defined and controlled, thus resulting in total 7 independent states. Two complex conjugate pole pairs show a nature similar to the stator and rotor eigenvalues. Study of these eigenvalues, as discussed below, provides insight about the nature of torque dynamics.

\subsection{Eigenvalue analysis}

Figure 12 shows the step response magnitude plot of $G_{s e c}$ for $3.73 \mathrm{~kW}$ machine along with the contributions by all eigenvalues and the direct term. Table 2 gives the numerical values of the $G_{\text {sec }}$ poles and direct term for $3.73,37.3$ and $373 \mathrm{~kW}$ machines for load torque values of 0.25 and 0.8 p.u. for the individual machines at a reference speed of 0.25 p.u., for MS and Mean control. From figure 12 it is clear that the real pole magnitude plays an important role in determining the speed of response of the transfer function. It can be observed that the magnitude of the real pole increases as the machine rating is higher, thus indicating a faster dynamic response for higher rated machines.

When the poles of $G_{s e c}$ and $G_{1}$ (or $G_{2}$ ) are compared, it can be observed that the real pole magnitude is much larger for $G_{1}$ poles thus making the dynamic response faster for the individual machines for the Mean control. It can also be observed that the direct term magnitudes are also higher in case of Mean control for all machine ratings. It can be clearly observed that 
Table 2. Transfer function eigenvalues.

\begin{tabular}{lccc}
\hline $\mathrm{kW}$ & 3.73 & 37.3 & 373.0 \\
\hline MS & $-200.12 \pm 69.41 i$ & $-188.99 \pm 24.39 i$ & $-54.46 \pm 53.10 i$ \\
Poles & $-5.21 \pm 69.65 i$ & $-4.11 \pm 50.16 i$ & $-2.985 \pm 31.48 i$ \\
& -1.56 & -9.39 & -26.95 \\
$k_{\text {sec }}$ & 1.926 & 3.292 & 16.25 \\
Mean & $-190.49 \pm 46.35 i$ & $-187.85 \pm 23.02 i$ & $-53.82 \pm 55.01 i$ \\
Poles & $-6.99 \pm 62.07 i$ & $-4.64 \pm 52.027 i$ & $-2.61 \pm 32.3 i$ \\
& $-7.74 \pm 18.43 i$ & $-6.22 \pm 5.81 i$ & $-1.236 \pm 1.97 i$ \\
& -21.13 & -11.196 & -29.07 \\
$k_{1}$ & 3.3082 & 3.474 & 17.55 \\
$k_{2}$ & 2.798 & 3.356 & 16.76 \\
\hline
\end{tabular}

the choice of control strategy affects the corresponding eigenvalues most significantly for 3.73 $\mathrm{kW}$ machines, whereas this effect is not as significant for higher rated machines. It can also be observed that this difference is the least for $373 \mathrm{~kW}$ machines, indicating that as the machine rating increases, the difference between the torque dynamics for the Mean and MS strategies becomes less significant.

\section{Experimental results}

To validate the analysis and simulations in the previous sections, a laboratory experimental setup has been assembled as shown in figure 1. The set-up consists of two nominally identical $3.73 \mathrm{~kW}, 415 \mathrm{~V}, 50 \mathrm{~Hz}$ induction machines coupled with dc generators for loading. They are fed by an IGBT inverter. The algorithms are implemented on a Digital Signal Controller (DSC) TMS320F28335. The line currents and speed feedback is taken from both machines for the implementation of the algorithm. The parameter values of the machines are as given in table 1 . Field oriented control using the MS and Mean strategies is implemented on the two-machine set-up in the laboratory. The nominal speed is 0.25 p.u. and the initial values of load torques on the two machines are $0.5 \mathrm{Nm}(0.034$ p.u.) and $4 \mathrm{Nm}$ (0.28 p.u.), respectively. To validate the dynamic performance analysis, a step change of $10 \%$ is given in the $q$ axis reference current. Figures 13(a)-14(a) show the superimposed plots of the torque difference obtained from numerical simulation as well as from the step response of the small-signal transfer function for the Primary and Secondary machines, respectively, for the same load and speed conditions. The torque difference is calculated by subtracting the initial value of torque from the actual torque after the step change in the reference. Figures 13(b)-14(b) show the experimental plots for the Primary and Secondary machine torque difference, for the MS control. For the Primary machine, it can be observed that the torque dynamic response is almost a step change, indicating a perfect field orientation. However, for the Secondary machine, the torque shows an oscillatory and delayed nature. It can also be observed that there is a close match between the values of torque obtained from the simulation and the small-signal analysis. The experimental torque plots for the Primary as well as Secondary machines show a similar trend as observed in the small-signal analysis plots. 


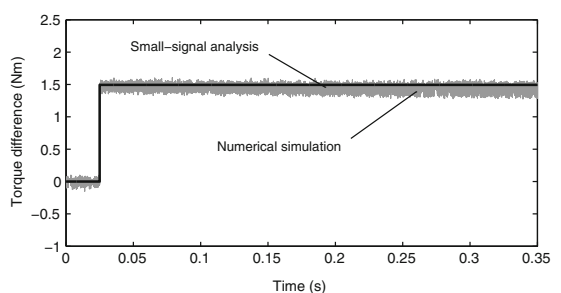

(a) Simulation and analysis

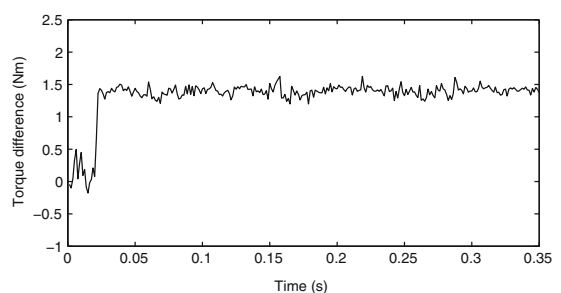

(b) Experimental

Figure 13. MS: Primary machine.

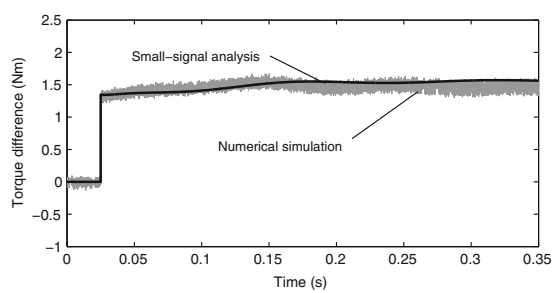

(a) Simulation and analysis

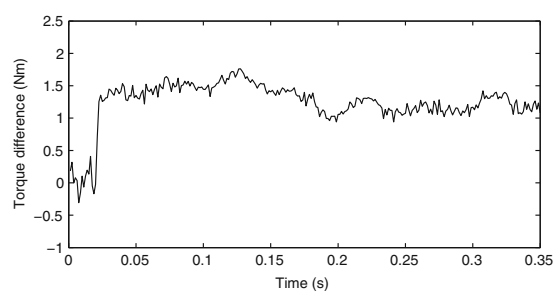

(b) Experimental

Figure 14. MS: Secondary machine.

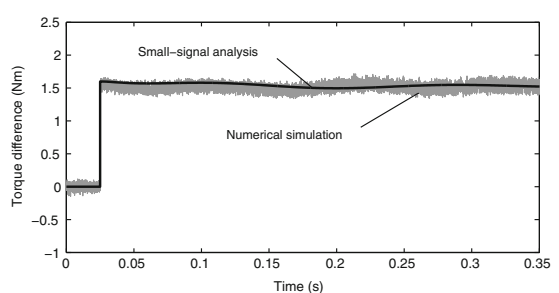

(a) Simulation and analysis

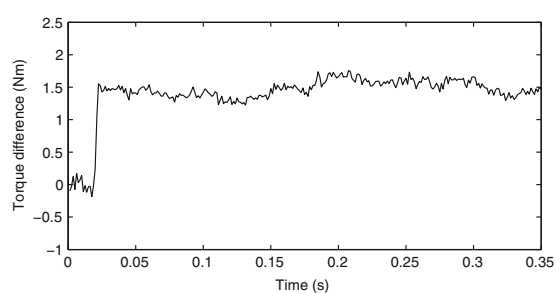

(b) Experimental

Figure 15. Mean: Machine 1.

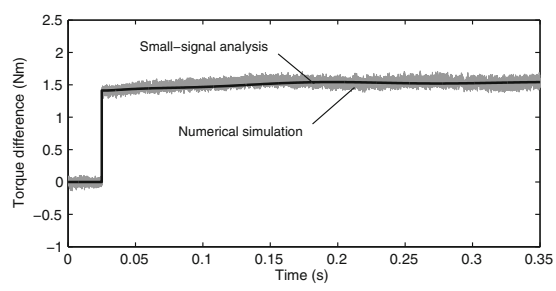

(a) Simulation and analysis

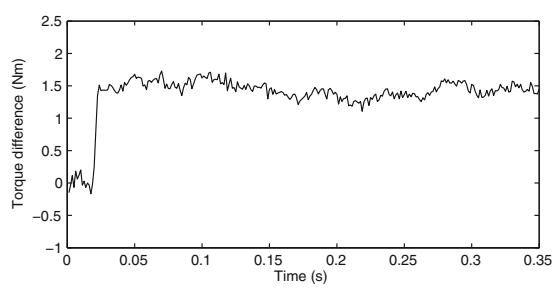

(b) Experimental

Figure 16. Mean: Machine 2. 
Figures 15(a)-16(a) show the superimposed plots of the torque difference obtained from simulation and small-signal analysis for machines 1 and 2 respectively, for the Mean control. Figures 15(b)-16(b) show the corresponding experimental plots. It can be observed that the torque dynamics of the individual machines show an oscillatory and delayed nature, however the dynamic response of the individual machines is faster as compared to that of the Secondary machine in the MS control. This nature is observed in the experimental plots as well.

\section{Conclusion}

In this paper, torque dynamics of two-machine single-inverter three-phase induction motor drives are studied and compared for the MS and Mean control strategies using small-signal analysis. For this purpose, a transfer function which relates the small-signal torque output to the smallsignal reference input is derived and its step-response is analysed. When the individual machine loads are different, while both machines' torque response shows a delayed and oscillatory nature in case of the Mean control, only the Secondary machine torque shows such behaviour in case of the MS control. However, the torque dynamics of Secondary machine in case of the MS control is inferior as compared to that of individual machines in case of the Mean control. This difference is more pronounced for drives with small machines. For higher rated machines, this difference is not significant. The study of eigenvalues of the small-signal transfer function while validating this analysis shows firstly that the choice of control strategy affects the torque response in a more significant manner for smaller machines as compared to larger machines and secondly, that the torque dynamic response becomes faster as the machine rating increases for a given control strategy. It can thus be concluded that the Mean control has a better overall dynamic response than the MS control, particularly for smaller rated machines and for a large load difference. The analysis is validated with numerical simulations and laboratory experiments.

\section{References}

Ando I, Sazawa M and Ohishi K 2004 High efficient speed control of parallel-connected induction motors with unbalanced load condition using one inverter. IECON 2: 1361-1366, 2-6

Bouscayrol A, Davat B, De Fornel B, Francois B, Hautier J P, Meibody-Tabar F and Pietrzak-David M 2000 Multi-machine multi-converter system for drives: Analysis of coupling by a global modeling. IEEE Industry Applications Conference 3: 1474-1481

Bouscayrol A, Delarue P, Fornel B D, Francois B, Hautier J P, Meibody-Tabar F, Monmasson E, Peitrzak-Davida M, Razik H, Semail E and Benkhoris M F 2005 Control structures for multi-machine multi-converter systems with several couplings by criteria merging. European Conference on Power Electronics and Applications, EPE

Crelerot O, Bernot F and Kauffmann J-M 1993 Study of an electrical differential motor for electrical car, Sixth International Conference on Electrical Machines and Drives. pp. 416-420

Escané P, Lochot C, David M and de Fornel B 1999 Electromechanical interactions in a high speed railway traction system comparison between two drive control structures. EPE Conference

Escané P, Pietrzak-David M and de Fornel B 2000 Optimization of a railway traction system drive control vs slip perturbation. Proc. of IEEE Industry Applications Conference 3: 1909-1916

Hartani K, Bourahla M, Miloud Y and Sekkour M 2008 Direct torque control of an electronic differential for electric vehicle with separate wheel drives. J Automation and Systems Eng. 2(2)

Inoue T, Ito S, Azegami K, Nakajima Y and Matsuse K 2011 Dynamic performance of sensorless vector controlled multiple induction motor drive connected in parallel fed by single inverter. IEEE Industry Applications Society Annual Meeting (IAS), pp. 1-6, 9-13 
Iyer J, Tabarraee K, Chiniforoosh S and Jatskevich J 2011 An improved V/F control scheme for symmetric load sharing of multi-machine induction motor drives, 24th Canadian Conference on Electrical and Computer Engineering (CCECE), pp. 1487-1490: 8-11

Jeftenic B, Bebic M and Statkic S 2006 Controlled multi-motor drives. International Symposium on Power Electronics, Electrical Drives, Automation and Motion, (SPEEDAM) 1392-1398: 23-26

Joshi B M and Chandorkar M C 2012 Power Failure Ride-through in Multi-machine Drives. In Proc. IEEE Energy Conversion Congress and Exposition (ECCE 2012) 15-20

Joshi B M and Chandorkar M C 2011 Effect of Machine Asymmetry on a Two-machine Direct Torque Controlled Induction Motor Drive. Proceedings of 14th European Conference on Power Electronics and Applications-EPE , Birmingham, UK, 30 Aug. -1

Joshi B M, Patel D C and Chandorkar M C 2011 Machine Interactions in Field Oriented Controlled MultiMachine Three Phase Induction Motor Drives. Proceedings of the IEEE International Electric Machines and Drives Conference (IEMDC), Niagara Falls, Canada, 15: 18

Kazmierkowski M P 1997 Control Philosophies of PWM Inverter-fed Induction Motors. Int. Conference on Industrial Electronics, Control and Instrumentation IECON '97, 1(P16-P26)

Kelecy P M and Lorenz R D 1994 Control methodology for single inverter, parallel connected dual induction motor drives for electric vehicles. In Proc. IEEE PESC'94, pp. 987-991

Krause P C, Wasynczuk O and Sudhoff S D 2002 Analysis of Electric Machinery and Drive Systems. New York: Wiley-Interscience

Levi E, Bojoi R, Profumo F, Toliyat H A and Williamson S 2007 Multiphase induction motor drives - a technology status review. IET Electric Power Applications, 1(4): 489-516

Levi E, Toliyat Jones M, Vukosavic S N and Toliyat H A 2004 A novel concept of a multiphase, multimotor vector controlled drive system supplied from a single voltage source inverter. In IEEE transactions on Power Electronics 19(2)

Ma J D, Bin Wu, Zargari N R and Rizzo S C 2001 A space vector modulated CSI-based AC drive for multimotor applications. IEEE Transactions on Power Electronics 16(4): 535-544

Matsumoto Y, Ozaki S and Kawamura A 2001 A novel vector control of single-inverter multiple-inductionmotors drives for Shinkansen traction system. 16th Annual Applied Power Electronics Conference and Exposition, APEC 1: 608-614

Matsuse K, Kouno Y, Kawai H and Yokomizo S 2002 A speed-sensorless vector control method of parallelconnected dual induction motor fed by a single inverter. IEEE Transactions on Industry Applications 38(6)

Mohktari H and Alizadeh A 2007 A new multi-machine control system based on Direct Torque Control algorithm. ICPE 1103-1108: 22-26

Novotny D W and Lipo T A 1996 Vector Control and Dynamics of AC Drives, Oxford University Press

Penã -Eguiluz R, Pietrzak-David M, Riga V and de Fornel B 2002 Comparison of several speed sensorless strategies of two different dual drive induction motor control structures. Power Electronics Congress. Technical Proceedings CIEP 2002. VIII IEEE International 41-46: 20-24

Ruxi W, Yue W, Qiang D, Yanhui H and Zhaoan W 2006 Study of Control Methodology for Single Inverter Parallel Connected Dual Induction Motors Based on the Dynamic Model. 37th IEEE Power Electronics Specialists Conference, PESC 1-7: 18-22

Tabbache B, Kheloui A and Benbouzid M E H 2011 An adaptive electric differential for electric vehicles motion stabilization. IEEE Transactions on Vehicular Technology, 60(1): 104-110

Taylor D G 1994 Nonlinear control of electric machines: An overview. IEEE Control Syst. Mag. 14: 41-51

Turl G, Summer M and Asher G M 2002 A Synchronised Multi-Motor Control System Using Sensorless Induction Motor Drives. International Conference on Power Electronics, machines and Drives pp. 3843: 4-7

Vas P 1998 Sensorless Vector and Direct Torque Control. New York: Oxford University Press 